\title{
Sex differences in early-life programming of the hypothalamic-pituitary-adrenal axis in humans suggest increased vulnerability in females: a systematic review
}

\author{
T. Carpenter, S. M. Grecian and R. M. Reynolds* \\ BHF Centre for Cardiovascular Science, Queen's Medical Research Institute, University of Edinburgh, Edinburgh, UK
}

\begin{abstract}
Fetal glucocorticoid overexposure is a key mechanism linking early development with later-life disease. In humans, low birth weight associates with increased fasting cortisol, hypothalamic-pituitary-adrenal (HPA) axis reactivity, and with cardiovascular risk and cognitive decline. As there are sex differences in these adult diseases, we hypothesized that there may be sex differences in programming of the HPA axis in response to prenatal stressors. We conducted a systematic review following Meta-Analysis of Observational Studies in Epidemiology and Preferred Reporting Items for Systematic Reviews and Meta-Analysis. We searched Embase, MEDLINE and Web of Science from inception to 31 October 2016. We included studies related to sex differences, prenatal exposures and HPA axis. We excluded studies investigating specific disease states. The 23 included studies investigated the consequences of low birth weight, preterm birth and maternal stressors of asthma, psychosocial stress and glucocorticoid medications on HPA axis outcomes of placental glucocorticoid biology and offspring HPA axis function in early life and later life. Female offspring exposed to stressors had increased HPA axis reactivity compared with males. Furthermore, the female placenta increased its permeability to maternal glucocorticoids following maternal stress with changes in the expression of $11 \beta$-hydroxysteroid dehydrogenase enzymes in response to maternal glucocorticoid exposure or asthma. Among males there was some evidence of altered diurnal cortisol secretion. We conclude that although there is some evidence of male vulnerability leading to altered diurnal cortisol secretion, the female HPA axis is more vulnerable to programming, particularly in terms of its reactivity; this suggests a mechanism underlying sex differences in later-life diseases.
\end{abstract}

Received 4 July 2016; Revised 25 November 2016; Accepted 27 November 2016; First published online 20 January 2017

Key words: human, HPA axis, sex differences

\section{Introduction}

Dysregulation of the hypothalamic-pituitary-adrenal (HPA) axis has been proposed as a key mechanism underlying the link between early-life development and later-life disease. ${ }^{1,2}$ Low birth weight, a surrogate marker of an adverse prenatal environment, is associated with higher fasting plasma cortisol, ${ }^{3}$ increased reactivity of the HPA axis ${ }^{4,5}$ and with cardiometabolic disease, mood disorders and accelerated cognitive decline. ${ }^{2}$ A number of studies have suggested that there may be sex differences in this response, but to our knowledge there has been no systematic review to establish whether this finding is consistent across all published studies. This may be an important consideration in the aetiology of later-life diseases, including depression and cardiometabolic disease, which show marked sex differences in presentation and prevalence. This systematic review of published observational studies aimed to determine whether there were sex differences in the HPA axis responses to prenatal stress in humans. Specifically, we aimed to determine whether there were sex-specific differences in placental handling of glucocorticoid hormones and thus fetal

*Address for correspondence: Professor R. Reynolds, BHF Centre for Cardiovascular Science, Queen's Medical Research Institute, University of Edinburgh, 47 Little France Crescent, Edinburgh EH16 4TJ, UK.

(Email r.reynolds@ed.ac.uk) glucocorticoid exposure and in HPA axis outcomes in the offspring at birth and across the lifespan.

\section{Methods \\ Data sources}

The Meta-Analysis of Observational Studies in Epidemiology guidelines were followed for conduct $^{6}$ and the Preferred Reporting Items for Systematic Reviews and Meta-Analysis (PRISMA) guidelines for reporting ${ }^{7}$ of this systematic review. The literature search was conducted using Embase, MEDLINE and Web of Science from inception to 31 October 2016. The searches were limited to human studies and used terms as both keywords and indexing terms (Medical Subject Headings, $\mathrm{MeSH}$ ) to identify studies related to sex differences, prenatal exposures and the HPA axis, for example, 'sex adj2 difference*', 'prenatal', 'cortisol' (for a full list, see Supplementary Table S1: 'Search Terms').

\section{Study selection}

All identified abstracts were screened for relevance by one reviewer (T.C.) and those that remained after screening were assessed by two reviewers (T.C. and S.M.G.) to establish whether they met the inclusion criteria. Any discrepancies in selection were discussed by all researchers until a consensus was reached. 
Studies were included if they investigated the prenatal environment, had a biological outcome that was related to the HPA axis (including placental handling of glucocorticoid hormones) and compared males and females. Studies were excluded if they investigated specific disease states where the disease does not reflect normal physiology (e.g. congenital adrenal hyperplasia, Cushing's disease). There were no exclusions related to study design.

\section{Data extraction}

Two independent reviewers (T.C. and S.M.G.) extracted relevant information on study design, characteristics, nature of prenatal exposure, methodology and outcome measures using a pre-specified data extraction form. Any disagreements were discussed and resolved by consensus.

\section{Quality assessment}

Studies were assessed for quality and risk of bias by the two reviewers (T.C. and S.M.G. or T.C. and R.M.R.) independently using a systematic scoring system ${ }^{8}$ with objective criteria relating to the clarity of the research question, participant recruitment and retention, reliability of exposure and outcome measures, and consideration of confounding variables (Supplementary Table S2: 'Quality Scoring'). A paper could attain a rating of 'high', 'intermediate' or 'low' with 80, 60 or $<60 \%$, respectively, of the 14 applicable criteria being satisfied.

\section{Data analysis and synthesis}

Due to considerable heterogeneity in both clinical characteristics, study design and data, a descriptive synthesis of results was conducted. This included a description of the study design and sample studied, details of the prenatal exposure, biological outcome measurement and whether there were sex differences in this outcome. Studies were grouped according to whether they (a) measured changes in genes regulating fetal glucocorticoid exposure in the placenta or (b) investigated HPA axis outcomes in the offspring at birth or longer-term follow-up. For presentation purposes we grouped the prenatal environmental exposures into maternal asthma, inhaled corticosteroids, antenatal corticosteroids, preterm birth, low birth weight and other forms of antenatal stress (e.g. maternal cortisol, subjective stress, significant life events).

\section{Results}

\section{Study design and participants}

From 173 titles and abstracts, 39 full-text articles were assessed, of which 23 were included in the final data synthesis, including data on 3739 participants (Fig. 1). The studies included 12 prospective cohort studies, nine cross-sectional studies and two case-control studies, and were conducted in Europe $(n=10)$, Australia $(n=7)$, United States $(n=3)$, South America

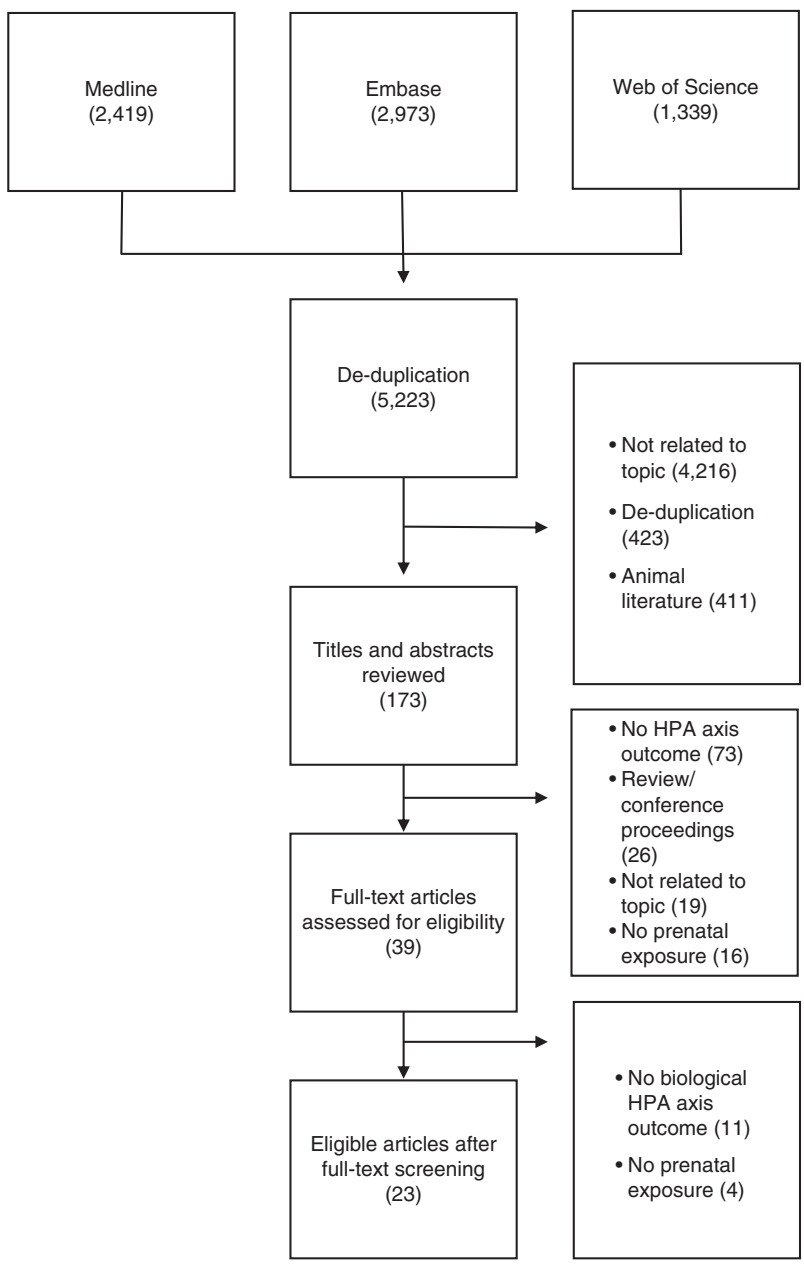

Fig. 1. Flow (Preferred Reporting Items for Systematic Reviews and Meta-Analysis) diagram of included studies. HPA, hypothalamicpituitary-adrenal.

$(n=2)$ and the Philippines $(n=1)$. Sample characteristics are detailed in Table 1.

\section{Studies investigating sex differences in placental glucocorticoid handling}

Eight studies investigated whether there were sex differences in genes regulating placental glucocorticoid handling and thus fetal glucocorticoid exposure (Table 2). The sample size varied from $43^{16}$ to $244^{20}$ placentas with exposures including maternal asthma, prenatal corticosteroids and preterm birth. Four studies investigated the effects of maternal glucocorticoid exposure on placental glucocorticoid receptor (GR) expression. ${ }^{12,17,25,28}$ Studies that investigated maternal asthma as a stressor identified sex differences, with females showing a decrease in GR messenger RNA compared with males ${ }^{17}$ and a different expression pattern of GR isoforms. ${ }^{25}$ One study found no sex-specific effect of inhaled forms of corticosteroids, except that males in the treatment group and the control group showed a positive correlation between placental GR 
Table 1. Descriptive table of all included articles

\begin{tabular}{|c|c|c|c|c|}
\hline Study & Year & Country & Study design & Sample characteristics \\
\hline Ballard et al. ${ }^{9}$ & 1980 & United States & Cross-sectional & 36 neonates ( 22 male, 14 female) \\
\hline Szathmari et al. ${ }^{10}$ & 2001 & Hungary & Cross-sectional & $\begin{array}{l}70 \text { young adults with low birth weight, mean age } 20 \text { years ( } 37 \text { male, } \\
33 \text { female), } 30 \text { controls with normal birth weight ( } 14 \text { male, } \\
16 \text { female) }\end{array}$ \\
\hline Fall et al. ${ }^{11}$ & 2002 & United Kingdom & Prospective cohort & 83 healthy adults, $61-72$ years ( 45 male, 38 female) \\
\hline Murphy et al. ${ }^{12}$ & 2003 & Australia & Prospective cohort & $\begin{array}{l}138 \text { neonates from mothers with asthma (62 mild, } 28 \text { moderate, } \\
48 \text { severe }^{\mathrm{a}} \text { ), } 44 \text { controls }\end{array}$ \\
\hline Reynolds et al. ${ }^{13}$ & 2005 & United Kingdom & Prospective cohort & 311 adults, mean age 71 years ( 205 male, 106 female) \\
\hline de Bruijn $e t$ al. ${ }^{14}$ & 2009 & The Netherlands & Cross-sectional & 103 children, $3-4$ years $(65$ male, 67 female $)$ \\
\hline Mericq et al. ${ }^{15}$ & 2009 & Chile & Cross-sectional & $\begin{array}{l}74 \text { placentas (32 from females, } 42 \text { from males), } 24 \text { SGA, } 25 \text { AGA, } \\
25 \text { LGA }\end{array}$ \\
\hline Stark et al. ${ }^{16}$ & 2009 & Australia & Case-control & 43 neonates and placentas ( 23 male, 20 female) \\
\hline Hodyl et al. ${ }^{17}$ & 2010 & Australia & Prospective cohort & $\begin{array}{l}122 \text { neonates and placentas from mothers with asthma ( } 52 \text { mild, } \\
\left.71 \text { moderate }^{\mathrm{b}}\right), 51 \text { controls; number of males and females } \\
\text { not stated }\end{array}$ \\
\hline $\begin{array}{l}\text { Osei-Kumah } \\
\quad \text { et al. }\end{array}$ & 2011 & Australia & Prospective cohort & $\begin{array}{l}49 \text { placentas, } 38 \text { from mothers with asthma ( } 23 \text { treated with GC, } \\
15 \text { not treated with GC), } 11 \text { controls ( } 22 \text { male, } 27 \text { female) }\end{array}$ \\
\hline Alexander et al. ${ }^{19}$ & 2012 & Germany & Cross-sectional & $\begin{array}{l}209 \text { children, } 6-11 \text { years ( } 113 \text { male, } 96 \text { female); } \\
81 \text { psychopathological pregnancies treated with GC, } \\
43 \text { psychopathological pregnancies not treated with GC, } \\
85 \text { controls }\end{array}$ \\
\hline Demendi et al. ${ }^{20}$ & 2012 & Hungary & Cross-sectional & 244 placentas, 104 preterm and 140 term ( 122 male, 122 female) \\
\hline Vedhara et al. ${ }^{21}$ & 2012 & United Kingdom & Prospective cohort & 139 adolescents, mean age 15 years ( 65 male, 74 female) \\
\hline Hodyl et al. ${ }^{22}$ & 2013 & Australia & Cross-sectional & $\begin{array}{l}53 \text { placentas and neonates with threatened preterm labour } \\
24-36 \text { weeks ( } 29 \text { male, } 24 \text { female) }\end{array}$ \\
\hline Lee $e t a l^{23}$ & 2014 & Philippines & Prospective cohort & $\begin{array}{l}1403 \text { young adults, } 21-23 \text { years ( } 802 \text { male, } 601 \text { female); } \\
215 \text { preterm, } 1188 \text { term }\end{array}$ \\
\hline Quesada et $a .^{24}$ & 2014 & Brazil & Cross-sectional & $\begin{array}{l}61 \text { children, } 6-10 \text { years ( } 32 \text { male, } 29 \text { female); } 30 \text { born preterm, } \\
31 \text { controls }\end{array}$ \\
\hline Saif et al. ${ }^{25}$ & 2014 & Australia & Prospective cohort & $\begin{array}{l}135 \text { neonates and placentas ( } 65 \text { male, } 70 \text { female); } 82 \text { mothers with } \\
\text { asthma, } 53 \text { controls }\end{array}$ \\
\hline Gohlke et al. ${ }^{26}$ & 2015 & Germany & Cross-sectional & $\begin{array}{l}54 \text { children, } 8-11 \text { years ( } 20 \text { male, } 34 \text { female); } 27 \text { with birth weight } \\
<1000 \text { g, } 27 \text { controls }\end{array}$ \\
\hline Mina et $a l_{.}^{27}$ & 2015 & United Kingdom & Case-control & $\begin{array}{l}93 \text { placentas ( } 34 \text { male, } 69 \text { female); } 50 \text { from severely obese mothers } \\
(\mathrm{BMI}>40), 43 \text { from lean mothers }(\mathrm{BMI}<25)\end{array}$ \\
\hline Saif $e t a l .{ }^{28}$ & 2015 & Australia & Prospective cohort & $\begin{array}{l}111 \text { placentas ( } 55 \text { male, } 56 \text { female); } 26 \text { preterm births treated with } \\
\text { GC, } 29 \text { preterm births not treated with GC, } 56 \text { controls }\end{array}$ \\
\hline Yong Ping et al. ${ }^{29}$ & 2015 & United States & Prospective cohort & $\begin{array}{l}94 \text { children, } 2.5 \text { years ( } 46 \text { male, } 48 \text { female), all exposed to prenatal } \\
\text { maternal stress }\end{array}$ \\
\hline Kaseva et al..$^{30}$ & 2016 & Finland & Prospective cohort & $\begin{array}{l}75 \text { young adults, mean age } 23.3 \text { years ( } 40 \text { male, } 35 \text { female); } 49 \text { with } \\
\text { very low birth weight, } 36 \text { controls }\end{array}$ \\
\hline Ostlund et al. ${ }^{31}$ & 2016 & United States & Prospective cohort & $\begin{array}{l}87 \text { infants, mean age } 19.8 \text { weeks ( } 42 \text { male, } 47 \text { female); } 41 \text { with } \\
\text { mothers who had a significant life event during pregnancy, } \\
46 \text { controls }\end{array}$ \\
\hline
\end{tabular}

SGA, small for gestational age; AGA, average size for gestational age; LGA, large for gestational age; GC, glucocorticoid medication, for example, betamethasone, dexamethasone; BMI, body mass index.

Articles ordered by year of publication.

${ }^{a}$ Asthma severity rated according to Australian Asthma Management guidelines.

${ }^{\mathrm{b}}$ Asthma severity rated according to Asthma Control Questionnaire (taking into account night waking, morning asthma symptoms, activity limitation, shortness of breath, wheezing, short-acting $\beta 2$-agonist use).$^{32}$ 
Table 2. Studies assessing placental outcomes

\begin{tabular}{|c|c|c|c|c|c|}
\hline Study & Sample characteristics & Exposure & Outcome measure & Sex difference & Findings \\
\hline Hodyl et al..$^{17}$ & $\begin{array}{l}\text { mRNA analysis: } 106 \text { placentas } \\
\text { (56 female, } 60 \text { male); } \\
83 \text { placentas from mothers } \\
\text { with asthma, } 33 \text { controls }\end{array}$ & Maternal asthma & $\begin{array}{l}\text { Placental GR } \\
\text { mRNA }\end{array}$ & Sex difference & $\begin{array}{l}\text { Decreased GR mRNA in females born to mothers with asthma (mild: } 0.4 \pm 0.35 \text {; } \\
\text { moderate-severe: } 0.75 \pm 0.25 \text { ), compared with control females }[1.55 \pm 0.4, F \\
(1,54)=4.99, P=0.003] \text {. No such change in GR mRNA expression in males } \\
\text { Data expressed as mean } \pm \text { S.E.M. Data points estimated from graph }\end{array}$ \\
\hline \multirow[t]{2}{*}{ Saif et al. ${ }^{25}$} & $\begin{array}{l}135 \text { neonates and placentas } \\
(65 \text { male, } 70 \text { female); } \\
82 \text { mothers with asthma, } \\
53 \text { controls }\end{array}$ & Maternal asthma & $\begin{array}{l}\text { Placental GR } \beta \\
\text { expression }\end{array}$ & Sex difference & $\begin{array}{l}\text { Increased cytoplasmic GR } \beta \text { in male placentas of pregnancies complicated by mild } \\
\quad \text { asthma [control: } 0.2(0.16-0.6), n=24 ; \text { remission: } 0.15(0.05-0.2), n=3 \text {; } \\
\text { intermittent: } 0.3(0.12-1.7), n=21 \text {; mild } 0.5(0.2-0.8), n=8 \text {; moderate- } \\
\text { severe: } 0.3(0.1-0.5), n=8] \\
\text { Data expressed as median (IQR) }\end{array}$ \\
\hline & & $\begin{array}{l}\text { Maternal asthma } \\
\text { and inhaled } \\
\text { corticosteroid } \\
\text { use }\end{array}$ & $\begin{array}{l}\text { Placental GR } \beta \\
\text { expression }\end{array}$ & Sex difference & $\begin{array}{l}\text { Decreased placental nuclear expression of GR } \beta \text { relative to cytoplasmic expression } \\
\text { in females }[0.33(0-1.1) v .0 .2(0-0.61), P=0.03, n=15] \\
\text { Data expressed as median (IQR) }\end{array}$ \\
\hline Saif $e t a l .{ }^{28}$ & $\begin{array}{l}111 \text { placentas ( } 55 \text { male, } \\
56 \text { female); } 26 \text { preterm births } \\
\text { treated with GC, } \\
29 \text { preterm births not treated } \\
\text { with GC, } 56 \text { controls }\end{array}$ & Preterm birth & $\begin{array}{r}\text { Placental GR } \\
\text { expression }\end{array}$ & Sex difference & $\begin{array}{l}\text { Increased cytoplasmic } \mathrm{GR} \alpha \text { isoform } \mathrm{C} \text { expression in male preterm placentas } \\
\text { relative to male term placentas [preterm: } 0.20(0-2.01) \text {; term: } 0(0-0.05) \text {, } \\
P=0.006]^{\mathrm{b}} \\
\text { Increased cytoplasmic GR A expression in male preterm placentas relative to male } \\
\text { term placentas [preterm: } 0.79(0-12.64) \text {; term: } 0(0-0), P=0.01 \text { ] } \\
\text { Increased nuclear GR } \alpha \text { isoform } \mathrm{C} \text { expression in female preterm placentas relative } \\
\quad \text { to term placentas [preterm: } 0.19(0-3.10) \text {; term: } 0(0-0.07), P=0.006 \text { ] } \\
\text { Data expressed as median (IQR) }\end{array}$ \\
\hline Murphy et al. ${ }^{12}$ & $\begin{array}{l}138 \text { neonates from mothers with } \\
\text { asthma }(62 \text { mild, } \\
\left.28 \text { moderate, } 48 \text { severe }^{\mathrm{c}}\right) \\
44 \text { controls }\end{array}$ & $\begin{array}{l}\text { Maternal asthma, } \\
\text { maternal } \\
\text { glucocorticoid } \\
\text { use }\end{array}$ & $\begin{array}{l}\text { Placental } 11 \beta- \\
\text { HSD2 activity }\end{array}$ & Sex difference & $\begin{array}{l}\text { Decreased placental } 11 \beta \text {-HSD2 activity in females from pregnancies complicated } \\
\text { by asthma whose mothers were not receiving glucocorticoid treatment } \\
(2.60 \pm 0.33 \mathrm{nmol} \text { cortisone } / \mathrm{mg} \text { protein } / \mathrm{h}, n=7) \text { compared with those } \\
\text { receiving glucocorticoids }(4.96 \pm 1.02 \mathrm{nmol} / \mathrm{mg} / \mathrm{h}, n=6) \text { and controls } \\
(6.88 \pm 0.59 \mathrm{nmol} / \mathrm{mg} / \mathrm{h}, n=26, P=0.002) \text {; no such difference in males } \\
\text { Decreased placental } 11 \beta \text {-HSD2 activity in placentas collected from males from } \\
\text { pregnancies complicated by asthma whose mothers were receiving } \\
\text { glucocorticoids compared with females from similar pregnancies }(P=0.0016) \\
\text { Data expressed as mean } \pm \text { S.E.M. }\end{array}$ \\
\hline Mericq et al. ${ }^{15}$ & $\begin{array}{l}74 \text { placentas ( } 32 \text { from females, } 42 \\
\text { from males), } 24 \text { SGA, } \\
25 \text { AGA, 25 LGA }\end{array}$ & $\begin{array}{l}\text { Reduced size for } \\
\text { gestational age } \\
\text { (SGA) }\end{array}$ & $\begin{array}{l}\text { Placental } 11 \beta- \\
\text { HSD2 activity }\end{array}$ & Sex difference & $\begin{array}{l}\text { Decreased placental } 11 \beta \text {-HSD2 activity in female SGA placentas compared with } \\
\text { male SGA placentas (female: } 5.2 \pm 0.9 \mathrm{pg} / \mathrm{min} / \mathrm{mg} ; \text { male: } 9.5 \pm \\
1.0 \mathrm{pg} / \mathrm{min} / \mathrm{mg}, P=0.007 \text { ) } \\
\text { Data expressed as mean } \pm \text { S.E.M. }\end{array}$ \\
\hline Stark et al. ${ }^{16}$ & $\begin{array}{l}43 \text { neonates and placentas } \\
(23 \text { male, } 20 \text { female })\end{array}$ & $\begin{array}{l}\text { Betamethasone } \\
\text { administered } \\
\text { either }<72 \mathrm{~h} \text { or } \\
>72 \mathrm{~h} \text { before } \\
\text { birth }\end{array}$ & $\begin{array}{l}\text { Placental } 11 \beta- \\
\text { HSD2 activity }\end{array}$ & Sex difference & $\begin{array}{l}\text { Increased placental } 11 \beta \text {-HSD2 activity rate in females compared with males when } \\
\text { betamethasone administered }<72 \mathrm{~h} \text { before delivery (female: } 619.2 \pm 70.8 \mathrm{nmol} / \\
\mathrm{mg} / \mathrm{min} \text {; male: } 313.4 \pm 46.4 \mathrm{nmol} / \mathrm{mg} / \mathrm{min}, P<0.01 \text { ) } \\
\text { Increased placental } 11 \beta \text {-HSD } 2 \text { total activity in females compared with males } \\
\text { when betamethasone administered }<72 \mathrm{~h} \text { before delivery (female: } \\
334.2 \pm 69.1 \mu \mathrm{mol} / \mathrm{h} \text {; male: } 121.3 \pm 38.1 \mu \mathrm{mol} / \mathrm{h}, P<0.01 \text { ) } \\
\text { Data expressed as mean } \pm \text { S.E.M. }\end{array}$ \\
\hline Demendi $e t a l .{ }^{20}$ & $\begin{array}{l}244 \text { placentas, } 104 \text { preterm and } \\
140 \text { term (122 male, } \\
122 \text { female) }\end{array}$ & Preterm birth & $\begin{array}{l}\text { Placental } 11 \beta- \\
\text { HSD2 gene } \\
\text { expression }\end{array}$ & No difference & $\begin{array}{l}\text { Decreased placental } 11 \beta \text {-HSD2 gene expression in preterm placentas compared } \\
\text { with term }[\alpha \text { value } \pm \text { s.E. }(\alpha),-2.7 \pm 0.83 \text {; lower confidence limit }-1.87 \text {, upper } \\
\text { confidence limit } 0.11, P=0.05]\end{array}$ \\
\hline
\end{tabular}


heterogeneous nuclear RNA and cord blood cortisol, but that there was no such association where the mothers had untreated asthma or the offspring was female. ${ }^{17}$ One study investigated the expression of GR isoforms in preterm birth, finding no sex differences in the total expression of GR apart from an increased level of GR $\alpha$ D2 in males. ${ }^{28}$ It did however find differences in GR localization, with preterm male placentas showing higher cytoplasmic concentrations of GR $\alpha \mathrm{C}$ and GR $\alpha$ A compared with full-term male placentas, with no such relationship in females. ${ }^{28}$

Four studies investigated effects on the expression and activity of $11 \beta$-hydroxysteroid dehydrogenase (11 -HSD) enzymes, with three of them finding a sex difference. With the exception of two studies, females showed changes that could lead to lower metabolism of cortisol by the placenta compared with males. This included reduced activity of placental $11 \beta-H S D 2$ with maternal asthma ${ }^{12}$ and prenatal corticosteroids, and with offspring reduced size for gestational age ${ }^{15}$ as well as increased activity of $11 \beta$-HSD1 with increased maternal cortisol. ${ }^{27}$ In contrast, one study found that $11 \beta-\mathrm{HSD} 2$ was increased in females following betamethasone exposure $>72 \mathrm{~h}$ before delivery. ${ }^{16}$ Another study found reduced placental $11 \beta-\mathrm{HSD} 2$ in both male and female preterm infants, with no sex difference. ${ }^{20}$

\section{Studies investigating sex differences in HPA axis outcomes in the offspring}

\section{Cord blood cortisol concentrations}

Six studies examined sex differences in umbilical cord blood cortisol concentrations with data from $n=527$ participants and prenatal exposures of maternal asthma ${ }^{12,17,25}$ and antenatal betamethasone for preterm birth ${ }^{9,16,22}$ (Table 3). Two studies found evidence of sex differences, with higher umbilical blood cortisol in females whose mothers had moderate or severe asthma compared with mild asthma ${ }^{17}$ and whose mothers were treated with betamethasone $<72 \mathrm{~h}$ before delivery. ${ }^{16}$ Two studies with maternal asthma ${ }^{12,25}$ and two with betamethasone $e^{9,22}$ found no sex differences in cord cortisol concentrations.

\section{Static HPA axis measures}

Five studies investigated plasma/serum cortisol concentrations (either in the morning or diurnal profiles), three with low birth weight $^{10,11,30}$, and two with preterm birth ${ }^{23,24}$, as the exposure (Table 3). The age at follow-up varied from 5 months to 72 years. One found that in childhood females born preterm had higher morning salivary cortisol than full-term females, as well as preterm and full-term males. ${ }^{24}$ One study in young adults found no difference in cortisol awakening response or diurnal cortisol secretion between individuals with very low birth weight and controls, with no sex differences. ${ }^{30}$ Another study in young adults found that males with preterm birth had decreased waking cortisol compared with males with term birth, and a shallower slope of diurnal cortisol decline, but found no such relationships among females. ${ }^{23}$ In contrast, a study in older 
Table 3. Offspring outcomes

\begin{tabular}{|c|c|c|c|c|c|}
\hline Study & Sample characteristics & Exposure & HPA axis measure & Sex difference & $\begin{array}{l}\text { Findings (mean cortisol in } \mathrm{nmol} / \mathrm{l} \text {, unless } \\
\text { otherwise specified) }\end{array}$ \\
\hline Ballard et al. ${ }^{9}$ & 36 neonates ( 22 male, 14 female) & $\begin{array}{l}\text { Betamethasone for } \\
\text { premature birth }\end{array}$ & Cord cortisol & No difference & $\begin{array}{l}\text { No difference between male and female cord cortisol } \\
\text { concentration, regardless of timing of betamethasone } \\
\text { administration [male: } 380 \text { (S.E.: 250); female: } 390 \\
\text { (S.E.: 190)] }\end{array}$ \\
\hline Murphy et al. ${ }^{12}$ & $\begin{array}{l}138 \text { neonates and placentas from } \\
\text { mothers with asthma }(62 \text { mild, } \\
28 \text { moderate, } 48 \text { severe }) \\
44 \text { controls }\end{array}$ & Maternal asthma & Umbilical vein cortisol & $\begin{array}{l}\text { No difference/ } \\
\text { difference not } \\
\text { tested }\end{array}$ & 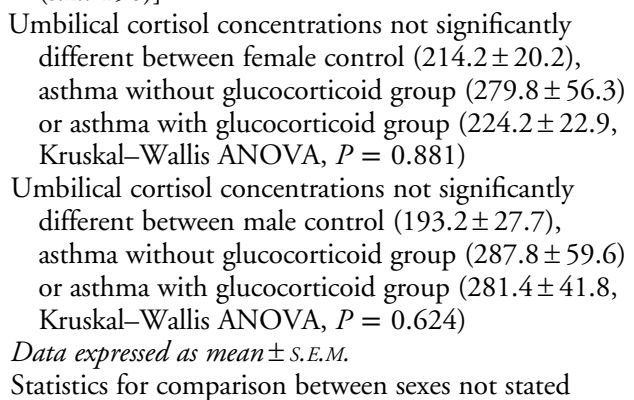 \\
\hline \multirow[t]{2}{*}{ Stark et al. ${ }^{16}$} & $\begin{array}{l}43 \text { neonates and placentas } \\
(23 \text { male, } 20 \text { female) }\end{array}$ & $\begin{array}{l}\text { Betamethasone for } \\
\text { premature birth, } \\
\text { administered } \\
<72 \mathrm{~h} \text { or }>72 \mathrm{~h} \\
\text { before delivery }\end{array}$ & Umbilical artery cortisol & Sex difference & $\begin{array}{l}\text { Increased female umbilical artery cortisol }(190 \pm 70) \\
\text { compared with male }(50 \pm 15) \text { when betamethasone } \\
\text { administered }<72 \mathrm{~h} \text { before delivery }[F(1,26)=7.59 \\
P=0.01] \text {, No difference when administered }>72 \mathrm{~h} \\
\text { before delivery }(P=0.14)\end{array}$ \\
\hline & & & $\begin{array}{l}\text { Day } 1 \text { neonatal urinary } \\
\text { cortisol (log nmol/l: } \\
\mathrm{mmol} / \mathrm{l} \text { of creatinine) }\end{array}$ & Sex difference & $\begin{array}{l}\text { Significant interaction between antenatal steroid } \\
\text { exposure, sex and perinatal stress }[F(1,38)=4.23 \text {, } \\
P=0.04] \text {. Increased female day } 1 \text { urinary cortisol } \\
(3.85 \pm 0.45) \text { compared with male }(1.8 \pm 0.45 \\
P<0.01) \text { when betamethasone administered }<72 \mathrm{~h} \\
\text { before delivery. No difference when administered } \\
>72 \mathrm{~h} \text { before delivery } \\
\text { Data expressed as mean } \pm \text { S.E.M. Data points estimated } \\
\text { from graph }\end{array}$ \\
\hline Hodyl et al. ${ }^{17}$ & $\begin{array}{l}122 \text { neonates and placentas from } \\
\text { mothers with asthma ( } 52 \text { mild, } \\
\left.71 \text { moderate }{ }^{\mathrm{b}}\right), 51 \text { controls; } \\
\text { number of males and females } \\
\text { not stated }\end{array}$ & Maternal asthma & Cord cortisol & Sex difference & $\begin{array}{l}\text { Increased female fetal cord cortisol with maternal } \\
\text { moderate-severe asthma when compared against } \\
\text { mild asthma or control }[F(2,67)=3.2, P=0.047] \text {. } \\
\text { No such difference in males }\end{array}$ \\
\hline Hodyl et al. ${ }^{22}$ & $\begin{array}{l}53 \text { placentas and neonates with } \\
\text { threatened preterm labour } 24- \\
36 \text { weeks ( } 29 \text { male, } 24 \text { female) }\end{array}$ & $\begin{array}{l}\text { Betamethasone for } \\
\text { premature birth, } \\
\text { administered } \\
<72 \mathrm{~h} \text { or }>72 \mathrm{~h} \\
\text { before delivery }\end{array}$ & Cord cortisol & No difference & $\begin{array}{l}\text { No difference between male and female cord cortisol } \\
\text { levels, regardless of timing of betamethasone } \\
\text { administration [female, }<72 \mathrm{~h}: 35.9(11.4-56.1) v \text {. } \\
>72 \mathrm{~h}: 21.25(13.5-35) ; \text { male, }<72 \mathrm{~h}: 21.2 \\
(16.2-42.1) v .>72 \mathrm{~h}: 27.9(22.6-53.7), P \text {-value not } \\
\text { stated] } \\
\text { Data reported as: median (25th-75th percentile). Cortisol } \\
\text { in } n g / m l\end{array}$ \\
\hline
\end{tabular}


Table 3. Continued

\begin{tabular}{|c|c|c|c|c|c|}
\hline Study & Sample characteristics & Exposure & HPA axis measure & Sex difference & $\begin{array}{l}\text { Findings (mean cortisol in } \mathrm{nmol} / \mathrm{l} \text {, unless } \\
\text { otherwise specified) }\end{array}$ \\
\hline Saif et al. ${ }^{25}$ & $\begin{array}{l}135 \text { neonates and placentas } \\
(65 \text { male, } 70 \text { female); } \\
82 \text { mothers with asthma, } \\
553 \text { controls }\end{array}$ & Maternal asthma & Cord cortisol & $\begin{array}{l}\text { Evidence of difference } \\
\text { inconclusive }\end{array}$ & $\begin{array}{l}\text { Decreased male cord cortisol with maternal asthma } \\
\text { compared with controls; no such finding in females. } \\
\text { Male with maternal asthma: } 1.72 \text { (S.E.M.: } 0.1 \text { ), } \\
\text { control: } 2.7 \text { (s.E.M.: 0.4); female with maternal } \\
\text { asthma: } 1.9 \text { (S.E.M.: 0.09), control 1.8 (S.E.M.: 0.19), } \\
P \text {-value not stated }\end{array}$ \\
\hline Ostlund et al. ${ }^{31}$ & $\begin{array}{l}87 \text { infants, mean age } 19.8 \text { weeks } \\
\text { ( } 42 \text { male, } 47 \text { female); } 41 \text { with } \\
\text { mothers who had a significant } \\
\text { life event during pregnancy, } \\
46 \text { controls }\end{array}$ & Maternal SLE & $\begin{array}{l}\text { Glucocorticoid receptor } \\
\text { gene }(N R 3 C 1) \\
\text { methylation }\end{array}$ & $\begin{array}{l}\text { Trend towards sex } \\
\quad \text { difference }\end{array}$ & $\begin{array}{l}\text { Increased methylation of } N R 3 C 1 \text { in females whose } \\
\text { mother had experienced an SLE during pregnancy } \\
(2.38, \text { s.D. }=0.99) \text { compared with controls } \\
(1.85, \text { s.D. }=0.41), t(21,41)=-2.01, P=0.057 \text {. } \\
\text { No such trend in males: } t(33)=-0.20, P=0.84\end{array}$ \\
\hline Gohlke et al. ${ }^{26}$ & $\begin{array}{l}54 \text { children, } 8-11 \text { years ( } 20 \text { male, } \\
34 \text { female); } 27 \text { with birth } \\
\text { weight }<1000 \mathrm{~g}, 27 \text { controls }\end{array}$ & ELBW $(<1000 \mathrm{~g})$ & $\begin{array}{l}\text { Urinary steroid } \\
\text { metabolites }\end{array}$ & $\begin{array}{l}\text { Sex difference not } \\
\text { tested }\end{array}$ & $\begin{array}{l}\text { Increased mean cortisol production in ELBW boys and } \\
\text { girls compared with controls. ELBW boys: } \\
12,400 \mu \mathrm{g} / \mathrm{l} \text {, control boys: } 6738 \mu \mathrm{g} / \mathrm{l}, P=0.05 \text {. } \\
\text { ELBW girls: } 10,717 \mu \mathrm{g} / \mathrm{l} \text {, control girls: } 6200 \mu \mathrm{g} / \mathrm{l} \text {, } \\
P=0.007\end{array}$ \\
\hline Szathmari et al. ${ }^{10}$ & $\begin{array}{l}70 \text { young adults with low birth } \\
\text { weight }(900-2500 \mathrm{~g}), \text { mean } \\
\text { age } 20 \text { years }(37 \text { male, } \\
33 \text { female), } 30 \text { controls } \\
\text { with normal birth weight } \\
\text { (14 male, } 16 \text { female) }\end{array}$ & Low birth weight & $\begin{array}{l}\text { Morning serum cortisol, } \\
\text { 7:30 am }\end{array}$ & No difference & $\begin{array}{l}\text { Females with low birth weight: } 250 \text { (s.D.: 70) } \\
\text { Males with low birth weight: } 270 \text { (s.D.: 60) } \\
\text { Statistics for comparison not stated }\end{array}$ \\
\hline Lee $e t a .^{23}$ & $\begin{array}{l}1403 \text { young adults, } 21-23 \text { years } \\
\text { (802 male, } 601 \text { female); } \\
215 \text { preterm, } 1188 \text { term }\end{array}$ & Preterm birth & $\begin{array}{l}\text { Diurnal salivary cortisol } \\
\text { profiles }\end{array}$ & Sex difference & $\begin{array}{l}\text { Decreased waking cortisol in males with preterm birth } \\
\text { compared with males with term birth. Term mean: } \\
7.2 \mathrm{mmol} / \mathrm{l}(\text { s.D.: } 4.5) \text {; preterm mean } 6.4 \mathrm{mmol} / \mathrm{l} \\
\text { (s.D.: } 3.3), P<0.05 \\
\text { No significant differences in cortisol in females with } \\
\text { preterm birth compared with females with term } \\
\text { birth. Waking cortisol, term mean: } 7.8 \mathrm{mmol} / \mathrm{l} \\
\text { (s.D.: 3.8); preterm mean: } 7.7 \mathrm{mmol} / \mathrm{l}(\text { s.D.: } 3.9) \\
\text { Slope of diurnal cortisol decline shallower in preterm } \\
\text { males, than term males. Term: }-0.32 \text { (s.D.: } \\
\text { 0.31) mmol////h; preterm: }-0.26 \text { (s.D.: } 0.26) \mathrm{mmol} / \mathrm{l} / \mathrm{h} \text {, } \\
P<0.05 \\
\text { No significant difference in slope of diurnal cortisol } \\
\text { decline in preterm females compared with term } \\
\text { females. Term: }-0.37 \text { (s.D.: } 0.25 \text { ) mmol////h; preterm: } \\
\text {-0.35 (s.D.: } 0.26 \text { ) mmol/l/h }\end{array}$ \\
\hline Fall et al. ${ }^{11}$ & $\begin{array}{l}83 \text { healthy adults, } 61-72 \text { years } \\
\text { ( } 45 \text { male, } 38 \text { female })\end{array}$ & Low birth weight & $\begin{array}{l}\text { Morning serum cortisol, } \\
\text { 7:30-9:00 am }\end{array}$ & $\begin{array}{l}\text { Evidence of difference } \\
\text { inconclusive }\end{array}$ & $\begin{array}{l}\text { Male: } 444.8 \text { (birth weight tertile 1), } 410.2,407.8 \text { (birth } \\
\text { weight tertiles } 2 \text { and 3); combined S.D.: } 90.1 \\
\text { Female: } 345.1 \text { (birth weight tertile 1) v. } 297.0,307.5 \\
\text { (birth weight tertiles } 2 \text { and 3); combined s.D.: } 79.4 \\
\text { Statistics for comparison not stated }\end{array}$ \\
\hline Yong Ping et al. ${ }^{29}$ & $\begin{array}{l}94 \text { children, } 2.5 \text { years ( } 46 \text { male, } \\
48 \text { female), all exposed to } \\
\text { prenatal stress }\end{array}$ & $\begin{array}{l}\text { Prenatal maternal } \\
\text { stress (Iowa flood } \\
\text { of 2008) }\end{array}$ & $\begin{array}{l}\text { Salivary cortisol following } \\
\text { mother-child } \\
\text { separation stressor }\end{array}$ & Sex difference & $\begin{array}{l}\text { Increased cortisol after stressor in females, no such effect } \\
\text { in males. Female cortisol increase } 20 \text { min post- } \\
\text { stressor: } t(40)=-2.019, P<0.05 ; 45 \text { min post- }\end{array}$ \\
\hline
\end{tabular}




(1)

de Bruijn et al. ${ }^{14}$

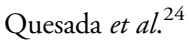

\begin{tabular}{|c|c|}
\hline Alexander et al..$^{19}$ & $\begin{array}{l}209 \text { children, } 6-11 \text { years } \\
\text { (113 male, } 96 \text { female); } 81 \\
\text { psychopathological } \\
\text { pregnancies treated with GC } \\
\text { (PP/GC), } 43 \\
\text { psychopathological } \\
\text { pregnancies not treated with } \\
\text { GC, } 85 \text { controls }\end{array}$ \\
\hline Vedhara et al. ${ }^{21}$ & $\begin{array}{l}139 \text { adolescents, mean age } \\
15 \text { years ( } 65 \text { male, } 74 \text { female) }\end{array}$ \\
\hline Kaseva et al. ${ }^{30}$ & $\begin{array}{l}75 \text { young adults, mean age } \\
23.3 \text { years ( } 40 \text { male, } \\
35 \text { female); } 49 \text { with VLBW, } \\
36 \text { controls }\end{array}$ \\
\hline
\end{tabular}

Prenatal maternal anxiety or affective complaints (scoring $>1$ S.D. over the mean on three separate prenatal anxiety and depression depression assessments) 61 children, $6-10$ years (32 male, 29 female); 30 born preterm, 31 controls

Course of

dexamethasone/ betamethasone in mothers admitted for complications; psychopathological pregnancy

Prenatal maternal depression

VLBW $(<1500 \mathrm{~g})$
Log score of salivary cortisol concentrations at the start of a home visit (T1), after mother-child interaction task (T2) and after a potentially frustrating task (T3)

\section{Awakening salivary} cortisol

Peak salivary cortisol concentrations following Trier Socia Stress Test (children)

Peak salivary cortisol

concentrations

following Trier Social Stress Test (children)

Log score of peak salivary cortisol following 35\% $\mathrm{CO}_{2}$ stress test

Log score of cortisol awakening response

Log score of diurnal cortisol secretion

Log score of cortisol following

dexamethasone suppression test stressor: $t(42)=-2.386, P<0.05$. Data for males not reported

Increased salivary cortisol after $45 \mathrm{~min}$ in females whose mothers reported greater subjective stress during pregnancy $(y=0.0464 x-0.0014, P<0.05)$; no such effect in males $(y=0.0179 x+0.0377, P=$ not significant). Increased area under the curve for cortisol after $45 \mathrm{~min}$ whose mothers reported greater subjective stress during pregnancy

$(y=0.0483 x-0.0721, P<0.05)$; no such effect in males $(y=-0.0108 x+0.0163 ; P=$ not significant $)$

Male: T1, 0.37 (s.D.: 0.29); T2, 0.21 (s.D.: 0.22); T3, 0.17 (s.D.: 0.25)

Female: T1, 0.60 (s.D.: 0.42); T2, 0.39 (s.D.: 0.29); T3, 0.30 (s.D.: 0.23 )
Sex difference

Sex difference

Sex difference

No difference

No difference

No difference
Higher awakening cortisol in preterm females (15.5, S.E.M.: 1.0) than full-term females (10.6, S.E.M.: 0.9, $P=0.040)$, preterm males (11.0, S.E.M.: 0.9) and full-term males (10.1, S.E.M.: 0.9, $P$-value not stated) Higher peak cortisol in preterm females (18.2, S.E.M.: 2.7) than full-term females $(6.0$, S.E.M.: 2.4 $P<0.01)$, preterm males (8.5, S.E.M.: 2.4 ) and fullterm males (7.0, S.E.M.: $2.5, P$-value not stated)

Where mothers had psychopathological pregnancy, higher peak cortisol in females prenatally treated with GC than males prenatally treated with GC. Female PP/GC: 14.166 (95\% CI 11.354, 16.977); male PP/ GC: 9.256 (95\% CI 7.436, 11.076), $P<0.05$

Female: $-0.14(95 \%$ CI $-0.31,0.04)$

Male: $-0.14(95 \%$ CI $-0.27,-0.01)$

No difference in cortisol awakening response between VLBW (2.0, S.D.: 1.9) and control groups (2.0, S.D.:

No difference in daily salivary cortisol between VLBW males and male controls $[8.8 \%(95 \% \mathrm{CI}-31.3$, 72.2), $P=0.71]$ or females and female controls [18.8\% (95\% CI $-11.8,60.0), P=0.25]$

No difference in daily salivary cortisol following lowdose dexamethasone between VLBW males and male controls $[-16.7 \%$ (95\% CI -68.3, 118.6), $P=0.7]$ or females and female controls

$[-9.7 \%(95 \%$ CI $-49.9,139.9), P=0.81]$ $1.9, P=0.85)$; no sex differences (test not reported) 


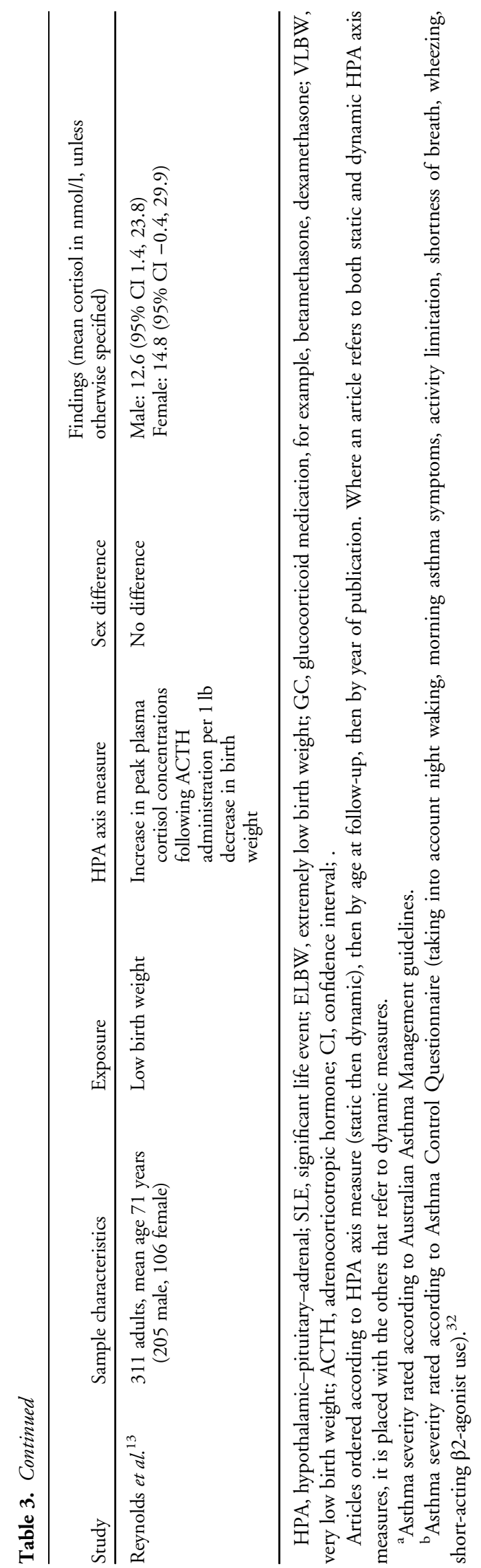

adults ${ }^{11}$ found higher morning serum cortisol concentrations in males and females of low birth weight than in males and females of higher birth weight but no formal statistical comparison was conducted. Two studies found increased morning salivary or serum cortisol in offspring exposed to neonatal corticosteroids or low birth weight, but found no sex differences in this effect either in early adulthood ${ }^{10}$ or adolescence. ${ }^{34}$ Another study investigated urinary steroid metabolites in extremely low birth weight children, with extremely low birth weight males and females having increased cortisol production compared with controls, but did not test whether there was any sex difference. ${ }^{26}$ A study investigating GR gene methylation in DNA extracted from buccal cells collected in infancy found that females whose mothers had experienced a significant life event during pregnancy had increased methylation of $N R 3 C 1$ compared with control females, finding no such relationship in males. ${ }^{31}$

\section{Dynamic HPA axis measures}

Six studies used a stress challenge to attempt to elicit differences in HPA axis reactivity (Table 3). Two used versions of the Trier Social Stress Test (TSST) were conducted in children and adolescents. ${ }^{19,24,34}$ Although the prenatal exposure differed (preterm birth; ${ }^{24}$ antenatal glucocorticoids ${ }^{19}$ ), both found a sex difference in HPA responses with a greater response in peak salivary cortisol in females. Two studies used other stressinducing tasks. One study among toddlers involved a brief period of separation from the child's mother and found that females had an increase in post-stressor cortisol and males did not, as well as a greater increase in cortisol in females whose mothers reported greater subjective stress during pregnancy, with no such correlation in males. ${ }^{29}$ Another study ${ }^{14}$ used a different stress-inducing task with 4-year-old children (confronting the child with a transparent box from which it was impossible to extract a toy), and found no difference in cortisol between males and females who had been exposed to prenatal maternal anxiety. One study used a physiological stressor a breath of $35 \%$ inhaled $\mathrm{CO}_{2}$ gas - in offspring exposed to maternal depression and found no sex differences in peak salivary cortisol. ${ }^{21}$

Neither of the studies testing HPA axis reactivity or central negative feedback [by adrenocorticotropic hormone (ACTH) stimulation or dexamethasone suppression, respectively] found sex differences in HPA axis responses to exposures of low birth weight ${ }^{13}$ or very low birth weight. ${ }^{30}$

\section{Quality assessment}

Most studies were determined to be of intermediate quality, scoring $60-80 \%$, with three being classified as low quality, scoring under $60 \%$. Studies scored as low quality either did not statistically adjust for confounding variables, did not report loss to follow-up, reported a loss to follow-up rate of over $20 \%$ or did not assess exposures more than once over time where it would have been applicable (Supplementary Table S2: 'Quality Assessment'). 


\section{Discussion}

This systematic review including 23 studies and data on $n=3739$ participants showed evidence of sex differences in HPA responses to prenatal stressors with increased vulnerability of the female HPA axis at a number of different levels and in response to a number of different prenatal stressors.

The placenta plays a key role in regulating fetal glucocorticoid exposure through the activity of the enzymes such as $11 \beta$-HSD2, which deactivates cortisol; $11 \beta$-HSD1, which regenerates cortisol; and the levels of GR, which signal glucocorticoid sensitivity. Overall, the findings suggested that in comparison with males, the female placenta increases its permeability to maternal glucocorticoids following maternal stress. This occurred through sexspecific lower expression of $11 \beta-H S D 2^{12}$ or increased expression of $11 \beta-\mathrm{HSD}^{27}$ and by changes in GR expression and localization, ${ }^{17,25,28}$ consistent with increased female fetal exposure to glucocorticoids. Having said this, one relatively large study that examined the association with preterm birth found a reduction in $11 \beta$-HSD2 in both sexes, with no sex difference, ${ }^{20,35}$ suggesting that in preterm birth at least, the nature of any sex difference is more complex. However, it may be the case that a dysfunctional placental $11 \beta$-HSD2 barrier is a causative factor in preterm birth itself, $^{20,35}$ and thus it is to be expected that preterm infants of both sexes show reduced placental $11 \beta$-HSD2.

When examining sex differences in HPA axis outcomes in the offspring, we identified studies using cord blood as an early measure of the infant HPA axis, and a number of studies measuring HPA axis activity in children and adults using both static and dynamic measures. Data on cord cortisol concentrations are difficult to interpret as they may represent either the basal level of secretion in the fetal HPA axis, the HPA axis response of the fetus to the stress of birth or short-term suppression of the fetal HPA axis by glucocorticoid medications. Two of the six identified studies ${ }^{16,17}$ reported higher cortisol concentrations in blood measured in female offspring, consistent with increased fetal glucocorticoid exposure in females. Findings from later in life were also not consistent. Studies on diurnal cortisol secretion either did not find a sex difference, ${ }^{10,26,30}$ found altered secretion in males ${ }^{23}$ or in females. ${ }^{24}$ Although the findings of HPA axis measures in the studies conducted in children and adults were not consistent, the only sex difference in HPA axis reactivity reported was consistent with increased reactivity in females. ${ }^{19,24,29}$ Of note, both of these studies were conducted using the TSST $^{19,24}$ and a maternal separation event, ${ }^{29}$ whereas other stress tests and hormone challenges failed to show any sex differences. ${ }^{13,14,21,30}$ The absence of findings from dexamethasone or ACTH administration suggests that the mechanism of sex-specific programming may be to do with higher neural inputs into the HPA axis rather than its sensitivity to its own components. However, one study suggested that sensitivity of the female physiology in general to glucocorticoids may be increased in situations of maternal stress. ${ }^{31}$

We included a broad definition of prenatal stressors including maternal inflammation, pharmacological intervention, psychological stressors and low birth weight/preterm birth. Low birth weight and preterm birth are considered the outcome of growth-restricting stressors during pregnancy and small for gestational age was associated with reduced activity of prenatal $11 \beta$-HSD2 and increased activity of $11 \beta$-HSD 1 in females. These changes would allow the delivery of more maternal cortisol to the fetus, and may indeed be a pathway contributing to growth restriction. Females with low birth weight or preterm birth had higher morning cortisol in one study, ${ }^{24}$ but not all. ${ }^{10,11,23,30}$ There was also evidence that females born preterm had increased HPA axis reactivity to stressful stimuli. ${ }^{24}$ Whether these changes are a cause or consequence of preterm birth is unknown. Nevertheless, if low birth weight and preterm birth are considered as a marker of prenatal stress, these findings support the argument that females are more vulnerable to the programming effects of prenatal stress in terms of HPA axis reactivity, possibly not in terms of diurnal secretion.

A number of studies considered maternal asthma as a prenatal stressor. Maternal asthma was found to decrease the placental barrier to glucocorticoids in terms of $11 \beta$-HSD $2^{12}$ and, putatively, GR. ${ }^{17,25}$ In one study, treatment of asthma with inhaled betamethasone ameliorated the effect, ${ }^{12}$ which suggests that it is the presence of the asthma itself rather than any associated factors in lifestyle or treatment that is responsible for the programming. However, it is possible that factors relating to treatment administration and compliance confound these results. No studies examined the association between maternal asthma and offspring later-life HPA axis outcomes, though these might be anticipated due to the observed alteration of the placental glucocorticoid barrier. A large follow-up study of clinical outcomes of offspring exposed to asthma in utero also did not examine whether there were sex differences in outcomes. ${ }^{36}$

Although use of prenatal glucocorticoids was associated with increased activity of placental $11 \beta-\mathrm{HSD} 2$ in females, it was also associated with an increase in cord blood cortisol in females when given within $72 \mathrm{~h}$ of delivery. ${ }^{16}$ Prenatal glucocorticoids were also associated with differential later-life outcomes, with females showing greater HPA axis reactivity to stress in terms of cortisol release. ${ }^{19}$ Overall, glucocorticoid medication appeared to induce offspring, although not placental, changes that were similar to those observed with inflammatory stress and low birth weight.

Our review identified studies investigating a variety of psychological stressors including maternal mood, ${ }^{21}$ emotional complaints, ${ }^{14}$ subjective stress ${ }^{29}$ and presence of psychopathology, ${ }^{19}$ but sex differences in the offspring HPA axis were only apparent in one study. ${ }^{29}$ This suggests that either the psychological measures studied were not severe enough to reliably induce a programming effect or that a biological/ hormonal response to stress - or its pharmacological mimic - is required to induce sex-specific prenatal programming. The one study that found a sex difference ${ }^{29}$ investigated mothers who had all been affected by a single major stressor - the Iowa flood of 2008 - suggesting that other studies investigated stressors whose nature was too broad, or not sufficiently severe. 
No studies investigated the association between maternal psychological measures and placental outcomes.

Mechanisms of the sex differences in the HPA axis responses to stress are unknown, but it is conceivable that they exist to effect the vulnerability-viability trade-off that is made by male and female fetuses in response to stress. It has been observed that males exposed to early environmental adversity suffer a risk to their viability. Although females suffer no such cost to their viability, they show increased vulnerability to adversity later in life. ${ }^{37}$

A strength of our study is that a systematic and comprehensive review process, devised with an experienced librarian, reported in line with PRISMA guidelines, was followed for this review. Two reviewers independently assessed eligibility of the titles, abstracts and full-text studies. Studies only including specific clinical populations with altered HPA physiology, for example, congenital adrenal hyperplasia, were excluded to ensure that our results were generalizable to the general population. However, there are some potential limitations to our study. The search terms were broad, and it is possible we have missed some potentially eligible studies. The studies were heterogeneous in terms of size, countries, ethnicities, age groups, methodology and reporting of statistical analysis. Most studies were graded as intermediate quality because they did not report sample size justification, participation rate of eligible persons, experimenter blinding or loss to follow-up.

In conclusion, although there was heterogeneity in study type and the nature of the findings, this systematic review demonstrated some evidence of increased programmed vulnerability to maternal stressors of the HPA axis in females compared with males, particularly in terms of HPA axis reactivity. Although there is evidence that males are not unaffected, any effect appears to be manifested in altered diurnal cortisol secretion. The limited evidence from diverse, moderate quality studies suggests that further research is required in order to determine whether this is a mechanism underlying differences in later-life diseases.

\section{Acknowledgements}

The authors thank Sheila Fisken, academic support librarian, for her help in designing the literature searches.

\section{Financial Support}

T.C. was supported by a College of Medicine and Veterinary Medicine summer vacation project bursary from the University of Edinburgh. R.M.R. acknowledges the support of Tommy's and the British Heart Foundation.

\section{Conflicts of Interest}

None.

\section{Supplementary material}

To view supplementary material for this article, please visit https://doi.org/10.1017/S204017441600074X

\section{References}

1. Edwards CR, Benediktsson R, Lindsay RS, Seckl JR. Dysfunction of placental glucocorticoid barrier: link between fetal environment and adult hypertension? Lancet. 1993; 341, 355-357.

2. Reynolds RM. Glucocorticoid excess and the developmental origins of disease: two decades of testing the hypothesis-2012 Curt Richter Award Winner. Psychoneuroendocrinology. 2013; 38, $1-11$.

3. van Montfoort N, Finken MJ, le Cessie S, Dekker FW, Wit JM. Could cortisol explain the association between birth weight and cardiovascular disease in later life? A meta-analysis. Eur J Endocrinol. 2005; 153, 811-817.

4. Reynolds RM, Walker BR, Syddall HE, et al. Altered control of cortisol secretion in adult men with low birth weight and cardiovascular risk factors. J Clin Endocrinol Metab. 2001; 86, 245-250.

5. Wust S, Entringer S, Federenko IS, Schlotz W, Hellhammer DH. Birth weight is associated with salivary cortisol responses to psychosocial stress in adult life. Psychoneuroendocrinology. 2005; 30, 591-598.

6. Stroup DF, Berlin JA, Morton SC, et al. Meta-analysis of observational studies in epidemiology: a proposal for reporting. Meta-analysis Of Observational Studies in Epidemiology (MOOSE) group. JAMA. 2000; 283, 2008-2012.

7. Liberati A, Altman DG, Tetzlaff J, et al. The PRISMA statement for reporting systematic reviews and meta-analyses of studies that evaluate healthcare interventions: explanation and elaboration. BMJ. 2009; 339, b2700.

8. National Institutes of Health NHLBI. Quality assessment tool for observational cohort and cross-sectional studies, 2014. Retrieved 4 January 2016 from http://www.nhlbi.nih.gov/health-pro/ guidelines/in-develop/cardiovascular-risk-reduction/tools/cohort.

9. Ballard PL, Ballard RA, Granberg JP. Fetal sex and prenatal betamethasone therapy. J Pediatr. 1980; 97, 451-454.

10. Szathmari M, Vasarhelyi B, Tulassay T. Effect of low birth weight on adrenal steroids and carbohydrate metabolism in early adulthood. Horm Res. 2001; 55, 172-178.

11. Fall CHD, Dennison E, Cooper C, et al. Does birth weight predict adult serum cortisol concentrations? Twenty-four-hour profiles in the United Kingdom 1920-1930 Hertfordshire Birth Cohort. J Clin Endocrinol Metab. 2002; 87, 2001-2007.

12. Murphy VE, Gibson PG, Giles WB, et al. Maternal asthma is associated with reduced female fetal growth. Am J Respir Crit Care Med. 2003; 168, 1317-1323.

13. Reynolds RM, Walker BR, Syddall HE, et al. Is there a gender difference in the associations of birthweight and adult hypothalamic-pituitary-adrenal axis activity? Eur J Endocrinol. 2005; 152, 249-253.

14. de Bruijn A, van Bakel HJA, Wijnen H, Pop VJM, van Baar AL. Prenatal maternal emotional complaints are associated with cortisol responses in toddler and preschool aged girls. Dev Psychobiol. 2009; 51, 553-563.

15. Mericq V, Medina P, Kakarieka E, et al. Differences in expression and activity of 11 beta-hydroxysteroid dehydrogenase type 1 and 2 in human placentas of term pregnancies according to birth weight and gender. Eur J Endocrinol. 2009; 161, 419-425.

16. Stark MJ, Wright IMR, Clifton VL. Sex-specific alterations in placental 11 beta-hydroxysteroid dehydrogenase 2 activity and 
early postnatal clinical course following antenatal betamethasone. Am J Physiol Regul Integr Comp Physiol. 2009; 297, R510-R514.

17. Hodyl NA, Wyper H, Osei-Kumah A, et al. Sex-specific associations between cortisol and birth weight in pregnancies complicated by asthma are not due to differential glucocorticoid receptor expression. Thorax. 2010; 65, 677-683.

18. Osei-Kumah A, Smith R, Jurisica I, Caniggia I, Clifton VL. Sex-specific differences in placental global gene expression in pregnancies complicated by asthma. Placenta. 2011; 32, 570-578.

19. Alexander N, Rosenlocher F, Stalder T, et al. Impact of antenatal synthetic glucocorticoid exposure on endocrine stress reactivity in term-born children. J Clin Endocrinol Metab. 2012; 97 , 3538-3544.

20. Demendi C, Borzsonyi B, Pajor A, et al. Abnormal fetomaternal glucocorticoid metabolism in the background of premature delivery: placental expression patterns of the 11 betahydroxysteroid dehydrogenase 2 gene. Eur J Obstet Gynecol Reprod Biol. 2012; 165, 210-214.

21. Vedhara K, Metcalfe C, Brant H, et al. Maternal mood and neuroendocrine programming: effects of time of exposure and sex. J Neuroendocrinol. 2012; 24, 999-1011.

22. Hodyl NA, Stark MJ, Butler M, Clifton VL. Placental P-glycoprotein is unaffected by timing of antenatal glucocorticoid therapy but reduced in SGA preterm infants. Placenta. 2013; 34, 325-330.

23. Lee J, Fried R, Thayer Z, Kuzawa CW. Preterm delivery as a predictor of diurnal cortisol profiles in adulthood: evidence from Cebu, Philippines. Am J Hum Biol. 2014; 26, 598-602.

24. Quesada AA, Tristao RM, Pratesi R, Wolf OT. Hyperresponsiveness to acute stress, emotional problems and poorer memory in former preterm children. Stress. 2014; 17, 389-399.

25. Saif Z, Hodyl NA, Hobbs E, et al. The human placenta expresses multiple glucocorticoid receptor isoforms that are altered by fetal sex, growth restriction and maternal asthma. Placenta. 2014; 35, 260-268.

26. Gohlke B, Wudy SA, Stutte S, et al. Increased steroid excretion in children with extremely low birth weight at a median age of 9.8 years. Horm Res Paediatr. 2015; 84, 331-337.
27. Mina TH, Raikkonen K, Riley SC, Norman JE, Reynolds RM. Maternal distress associates with placental genes regulating fetal glucocorticoid exposure and IGF2: role of obesity and sex. Psychoneuroendocrinology. 2015; 59, 112-122.

28. Saif Z, Hodyl NA, Stark MJ, et al. Expression of eight glucocorticoid receptor isoforms in the human preterm placenta vary with fetal sex and birthweight. Placenta. 2015; 36, 723-730.

29. Yong Ping E, Laplante DP, Elgbeili G, et al. Prenatal maternal stress predicts stress reactivity at $21 / 2$ years of age: the Iowa Flood Study. Psychoneuroendocrinology. 2015; 56, 62-78.

30. Kaseva N, Pyhala R, Pesonen AK, et al. Diurnal cortisol patterns and dexamethasone suppression test responses in healthy young adults born preterm at very low birth weight. PLoS ONE. 2016; 11, e0162650.

31. Ostlund BD, Conradt E, Crowell SE, et al. Prenatal stress, fearfulness, and the epigenome: exploratory analysis of sex differences in DNA methylation of the glucocorticoid receptor gene. Front Behav Neurosci. 2016; 10, 147.

32. Juniper EF, O’Byrne PM, Guyatt GH, Ferrie PJ, King DR. Development and validation of a questionnaire to measure asthma control. Eur Respir J. 1999; 14, 902-907.

33. Juniper EF, O’Byrne PM, Ferrie PJ, King DR, Roberts JN. Measuring asthma control. Clinic questionnaire or daily diary? Am J Respir Crit Care Med. 2000; 162(Pt 1), 1330-1334.

34. ter Wolbeek M, Kavelaars A, de Vries WB, et al. Neonatal glucocorticoid treatment: long-term effects on the hypothalamuspituitary-adrenal axis, immune system, and problem behavior in 1417 year old adolescents. Brain Behav Immun. 2015; 45, 128-138.

35. Johnstone JF, Bocking AD, Unlugedik E, Challis JRG. The effects of chorioamnionitis and betamethasone on $11 \beta$, hydroxysteroid dehydrogenase types 1 and 2 and the glucocorticoid receptor in preterm human placenta. J Soc Gynecol Investig. 2005; 12, 238-245.

36. Tegethoff M, Olsen J, Schaffner E, Meinlschmidt G. Asthma during pregnancy and clinical outcomes in offspring: a national cohort study. Pediatrics. 2013; 132, 483-491.

37. Sandman CA, Glynn LM, Davis EP. Is there a viabilityvulnerability tradeoff? Sex differences in fetal programming. J Psychosom Res. 2013; 75, 327-335. 